\title{
Development of microparticles from wheat glutenins by electrospray and potential application as controlled-release fertilizers
}

\author{
D-D CASTRO-ENRÍQUEZ ${ }^{1}$, M-M CASTILLO-ORTEGA ${ }^{1}$, J ROMERO-GARCÍA ${ }^{2}$, \\ D-E RODRÍGUEZ-FÉLIX ${ }^{1}$, R-F DÓRAME-MIRANDA ${ }^{1}$, W TORRES-ARREOLA ${ }^{3}$, \\ J-M VARGAS-LÓPEZ ${ }^{3}$, S-E BURRUEL-IBARRA ${ }^{1}$ and F RODRÍGUEZ-FÉLIX ${ }^{3, *}$ (i) \\ ${ }^{1}$ Departamento de Investigación en Polímeros y Materiales, Universidad de Sonora, Encinas y Rosales, 83000 Hermosillo, \\ Sonora, Mexico \\ ${ }^{2}$ Centro de Investigación en Química Aplicada, 25294 Saltillo, Coahuila, Mexico \\ ${ }^{3}$ Departamento de Investigación y Posgrado en Alimentos, Universidad de Sonora, Encinas y Rosales, 83000 Hermosillo, \\ Sonora, Mexico \\ *Author for correspondence (rodriguez_felix_fco@hotmail.com)
}

MS received 10 April 2018; accepted 6 August 2018; published online 7 February 2019

\begin{abstract}
Development of microparticles based on natural polymers has been of interest for researchers due to their applications, such as release systems. Currently, one of the problems presented by agriculture worldwide is the loss of fertilizers, i.e., urea, causing environmental pollution and high costs. The aim of this work was to develop microparticles of wheat glutenins by means of electrospray technique, with potential application as a urea controlled-release system in agricultural soils. The microparticles of wheat glutenins were characterized by scanning electron microscopy, Fourier transform infrared spectroscopy (FT-IR) and thermogravimetric analysis (TGA). In addition, a release kinetic test was performed to evaluate the possible behaviour of wheat-glutenin microparticles in agricultural soils with $\mathrm{pH} 4,7$ and 10 , using buffers as the release medium. TGA indicated that microparticle stability was $>100^{\circ} \mathrm{C}$, while FT-IR demonstrated the existence of physical interactions between urea and wheat glutenins. The kinetic tests showed the possible behaviour of the controlled-release fertilizer at $\mathrm{pH} \mathrm{4,7}$ and 10; rapid release at acidic $\mathrm{pH}$ and a decrease in release time at basic $\mathrm{pH}$. With these results, we can conclude that the urea-charged wheat-glutenin microparticles can function as a controlled-release fertilizer in agricultural soils.
\end{abstract}

Keywords. Microparticles; glutenins; electrospray; controlled-release fertilizer.

\section{Introduction}

Many global activities have been promoted for environmental conservation and protection, generating the study, production and use of several natural polymers for the development of materials with applications in foods, medicine, agriculture, etc. $[1,2]$. Cereal proteins are natural polymers that offer interesting possibilities for developing new materials, due to their biodegradability, low cost, availability, cyto-biocompatibility and the presence of functional groups which can interact with substances-of-interest or that can be modulated in response to changes in $\mathrm{pH}$. In addition, cereal proteins such as zein and wheat gluten are generated as by-products when the grains are processed for production of food or biofuels [3].

Gluten proteins, specifically glutenins, are promising for the development of new materials, because the materials obtained from glutenins possess better physicochemical properties compared with materials of zein and gliadin. Additionally, glutenins do not have immune responses to persons with celiac disease $[3,4]$. Glutenins are polymeric proteins of high-molecular weight (HMW), ranging from 32,000 to more than 10 million Da; they have disulphide intermolecular and intramolecular bonds between their chains, and they present two types of subunits: HMW and low-molecular weight (LMW). These subunits can be obtained from reducing the disulphide bonds of glutenin polymers, using reducing agents, and these are practically soluble in diluted acid solutions $[5,6]$. To date, the materials obtained from glutenins are limited, due to difficulty in processing that is attributed to their highly cross-linked molecular structure, demonstrating a challenge in obtaining materials. Despite difficulty in obtaining materials from glutenins, in the literature, it has been reported that nano- and microfibres have been obtained by electrospinning and films have been obtained by several methods [7-9]. Recently, Reddy et al [10] obtained nanoparticles from glutenins, evidencing that these are suitable for use in drug release.

Over the last 10 years, the development of microparticles based on natural and synthetic polymers has attracted great interest for numerous applications, mainly as release 
systems [11]. Microparticles offer a longer diffusional path, which usually leads to slower release [12]. In the literature, there are several methods available for the production of microparticles, e.g., emulsions, interfacial polymerization, nanoprecipitation and spray drying. Their disadvantages are that they are time-consuming and there is prolonged contact with organic solvents, causing denaturation of molecules that require encapsulation $[11,13]$. The electrospray technique can generate nano- and microparticles in an easy and economical manner, and can be used at an industrial scale, using a series of atomizers [14-17]. This technique generates small drops by applying an electric field. In this process, the liquid flowing out from a capillary is maintained at high potential, which gives rise to the elongation of the meniscus, breaking into fine drops [18].

One potential application of these systems of microparticles is in agriculture, where the main problem presented is the loss of fertilizers, such as nitrogen $(\mathrm{N})$ in the form of urea. Urea is the most inexpensive nitrogenous fertilizer and it is used in agricultural production worldwide. When urea is applied to agricultural crops, losses are produced up to $90 \%$ due to leaching, volatilization and denitrification, affording environmental impacts, low yields, poor product quality and high costs to the farmer $[3,5,19]$. Hence, there is an urgent need to generate new investigations and technologies, which allows preservation of the environment, achieving maximal production potential and reducing agricultural costs. The aim of this work was to obtain wheat-glutenin microparticles by means of the electrospray technique and their study as a potential urea controlled-release system with application in agricultural soils.

\section{Experimental}

\subsection{Materials and methods}

The material included is commercial wheat gluten, Roquette brand (France). Its proximal composition was as follows: moisture content, 3.9\%; protein content, $74.0 \%$; ash, $2.0 \%$ and fat, $2.1 \%$. Also, the reagents used were ethanol (99.9\% purity; J. T. Baker), glacial acetic acid (99.5\% purity; Faga Lab), sodium sulphite $\left(\mathrm{Na}_{2} \mathrm{SO}_{3}\right.$, Fermont), urea (99.2\% purity, Fermont) and sodium dodecyl sulphate (SDS; Bio-Rad), and a urease Berthelot kit (Randox) was used. All reagents were used as received.

\subsection{Extraction of wheat glutenins}

Glutenins were obtained from $100 \mathrm{~g}$ of commercial wheat gluten and dispersed in $1600 \mathrm{ml}$ of ethanol (70\%), and then stirred for $12 \mathrm{~h}$ at $25^{\circ} \mathrm{C}$ and centrifuged at $5000 \times g$ for $15 \mathrm{~min}$ at $25^{\circ} \mathrm{C}$. Subsequently, the precipitate (glutenins) was washed with distilled water to remove the remaining ethanol, and then, it was frozen, lyophilized and ground with a porcelain mortar and pestle.

\subsection{Chemical treatment of glutenins}

Glutenins were fractionated to obtain the soluble fraction of glutenins (SFG) using the modified methodology of $\mathrm{Xu}$ et al [9]. A $13 \%$ solution (w/v) of wheat glutenins was prepared and the following solutions were added: $8 \mathrm{M}$ urea; $2: 1$ acetic acid (70\%); $\mathrm{Na}_{2} \mathrm{SO}_{3}(2 \%)$ and $\mathrm{SDS}(4 \%)$. The solution was placed under magnetic stirring at $40^{\circ} \mathrm{C}$ for $24 \mathrm{~h}$ and was then centrifuged at $8000 \times g$ for $20 \mathrm{~min}$. The supernatant was dialysed in a cellulose membrane with a pore size of $14,000 \mathrm{Da}$ and was then frozen, lyophilized and ground with a porcelain mortar and pestle.

\subsection{Preparation of microparticles by electrospray}

The solutions were prepared from SFG at different concentrations of 4, 12 and $20 \%(\mathrm{w} / \mathrm{v})$ utilizing acetic acid (80\%) diluted with $1 \mathrm{M} \mathrm{Na}_{2} \mathrm{SO}_{3}$ and SDS (4\%). Conditions for the electrospray process were established according to the macroscopic characteristics of the material obtained. The solutions were poured into a syringe with a 1-ml capacity. The conditions used were as follows: a needle of $0.30-\mathrm{mm}$ in diameter; distance between capillary and the collecting plate $(15-20 \mathrm{~cm})$; flow rate $\left(0.1-1.2 \mathrm{ml} \mathrm{h}^{-1}\right)$; voltage $(15-20 \mathrm{kV})$ and positive potential.

The best solution of SFG to obtain microparticles was added to $1.20 \mathrm{~g}$ of urea, and this was stirred for $10 \mathrm{~min}$. Subsequently, microparticles were obtained with urea by electrospray from the optimal conditions.

\subsection{Load amount of urea}

Loading efficiency was obtained by weighing $180 \mathrm{mg}$ of microparticles loaded with urea and scattered in a buffer at $\mathrm{pH}$ 7. The urea concentration was determined using a colorimetric method with the Randox kit. A $10 \mu 1$ of sample was mixed with $1000 \mu \mathrm{l}$ of phosphate buffer and urease, and was incubated for $5 \mathrm{~min}$ at $25^{\circ} \mathrm{C}$. Subsequently, $200 \mu \mathrm{l}$ of sodium hypochlorite was added, mixed and incubated for $10 \mathrm{~min}$ at $25^{\circ} \mathrm{C}$, obtaining a green colouration. Absorbance was measured at $700 \mathrm{~nm}$ in an ultraviolet (UV)-Vis spectrophotometer (Perkin-Elmer; Lambda 20). The amount of urea in the sample was calculated from the calibration curve previously prepared with buffer at $\mathrm{pH}$ 7. The amount of filler was determined by the ratio by the weight contained in the microparticles and that was used in the preparation of the solution [12].

\subsection{Determination of molecular weight by SDS-PAGE}

The solution was prepared with $120 \mathrm{mg}$ of SFG and $1 \mathrm{ml}$ of acetic acid $(80 \%)$ diluted with $1 \mathrm{M} \mathrm{Na}_{2} \mathrm{SO}_{3}$ and SDS. This was centrifuged at $8000 \mathrm{rpm}$ for $5 \mathrm{~min}$ and the supernatant was used to obtain a 1:10 dilution with buffer Tris- $\mathrm{HCl}$ at $\mathrm{pH}$ 8.8. To this dilution, we added 2-mercaptoethanol 1:1; this was heated for $5 \mathrm{~min}$ and then cooled. A $20 \mu \mathrm{l}$ of sample was injected into a polyacrylamide gel (10\%) at 100 V. For 
revealing the bands, silver staining was used. To obtain the image, we employed Quantity One-4-6-9 software (Bio-Rad).

\subsection{Rheological analysis}

Anton Paar rheometer equipment (MCR; model 102) with concentric cylinder geometry was utilized to study the rheological properties of the solutions. The solutions were analysed at a constant shear rate of $5 \mathrm{~s}^{-1}$, which was obtained from scanning the shear rate within a range of $0.1-20 \mathrm{~s}^{-1}$. All determinations were carried out in triplicate.

\subsection{Morphological studies}

Morphological characteristics of the microparticles were evaluated using a scanning electron microscope (SEM), JEOL brand, model $5410 \mathrm{LV}$, operated at $15 \mathrm{kV}$. Samples were goldcoated before their analysis.

\subsection{FT-IR analysis}

FT-IR spectroscopy was performed using Perkin-Elmer GX equipment. A $5 \mathrm{mg}$ of sample was embedded in a $\mathrm{KBr}$ pellet. The number of scans was 16 within a range from an IR of $4000-500 \mathrm{~cm}^{-1}$.

\subsection{Thermal analysis}

Thermal stability of the materials was determined employing a Perkin-Elmer Thermogravimetric Analyzer, model Pyris 1. A $4 \mathrm{mg}$ of sample was weighed and heated to $700^{\circ} \mathrm{C}$ with heating and cooling rates of $10^{\circ} \mathrm{C} \mathrm{min}^{-1}$, under a nitrogen

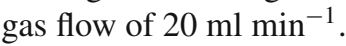

\subsection{Release kinetics urea}

Kinetics of urea release were made from tablets; $180 \mathrm{mg}$ of urea-loaded microparticles were weighed and placed in a mould with dimensions of $1.3 \mathrm{~cm}$ in diameter and $3 \mathrm{~mm}$ in thickness, pressed at $160 \mathrm{MPa}$ in a Carver press (C1). The tablets were then soaked in buffers at $\mathrm{pH} 4,7$ and 10 at $25^{\circ} \mathrm{C}$ and were subsequently placed in a shaker incubator (Barnstead; Max Q 4000). Then, a $10 \mu \mathrm{l}$ of sample was taken at different times. The released urea concentration was determined using a colorimetric method (Randox kit, methodology described previously). Samples were read at a wavelength of $700 \mathrm{~nm}$ in a UV-Vis spectrophotometer (Perkin-Elmer; Lambda 20) and collected until the concentration within the solution remained constant. All experiments were performed in quadruplicate.

\subsection{Empirical model}

To determine the release mechanism of urea at $\mathrm{pH} \mathrm{4,7} \mathrm{and}$ 10, the model of Ritger and Peppas was chosen, which is represented by the following equation:

$$
M_{\mathrm{t}} / M_{\infty}=k t^{n}
$$

where $M_{\mathrm{t}} / M_{\infty}$ is the fraction of urea released at time $t, k$ is a kinetic constant and $n$ is the exponent of diffusion indicative of the transport mechanism of urea through the microparticle. Values of $n$ for thin matrices are: when $n=0.5$ release mechanism of urea is by Fickian diffusion; when $n=1$, the transport that occurs is case-type II or zero-order and when $n$ is from $0.5-1$, it is an anomalous mechanism [20,21].

\section{Results and discussion}

\subsection{Determination of molecular weight by SDS-PAGE}

Figure 1 shows the molecular weight of the SFG used in this study under reducing conditions. It exhibits an intense band around $58 \mathrm{kDa}$. This band is attributed to subunits of HMW glutenin (HMW-G), considered proteins of relatively LMW [22]. Gómez et al [15] reported nanoparticles of zein by means of the electrohydrodynamic atomization technique, with lower molecular weights (25-30 kDa) compared with those obtained in this study. This difference in the molecular weight of cereal proteins could exert an influence on obtaining nanoparticles or microparticles. Therefore, we concluded that to obtain wheat-glutenin microparticles by the electrospray technique, a molecular weight of $58 \mathrm{kDa}$ is required.

\subsection{Rheological analysis}

Polymer concentration is an important factor to obtain nanoor microparticles by the electrospray technique, where viscosity affects the size and morphology of particles [3]. Therefore, an increase or decrease in viscosity determines the physical characteristics of the particles. It is known that to obtain particles by electrospray, low concentrations of the polymer are required, but this depends on their molecular weight. The literature indicates that the viscosity value limit for particle formation is $800 \mathrm{mPa} \mathrm{s}^{-1}$ [23]. Thus, solutions with viscosities below this limit are diluted ones that will tend to disintegrate into drops.

Figure 2 depicts the dependence of viscosity with respect to the increase in shear rate for solutions of 4, 12 and $20 \%$. The $4 \%$ solution revealed no effect in terms of viscosity and an increase in shear rate, i.e., viscosity is stable regardless of the shear rate. This behaviour is attributed to the fact that the solutes have better dissolution in the solvent, which causes the solution to be stable [24,25]. However, as the solute concentration increases (12 and 20\%), the viscosity is unstable with respect to a higher shear rate. Therefore, this indicates that the solutes are not completely dissolved in the solution, hence, obtaining a change in viscosity. This behaviour can be confirmed by knowing the fluid type for each concentration of the solutions (table 1). The $4 \%$ solution is a Newtonian solution; thus, it obeys Newton's law, being a stable solution without a change in viscosity. Solutions of higher concentrations (12 and $20 \%$ ) exhibit pseudoplastic behaviour. This is due to the 


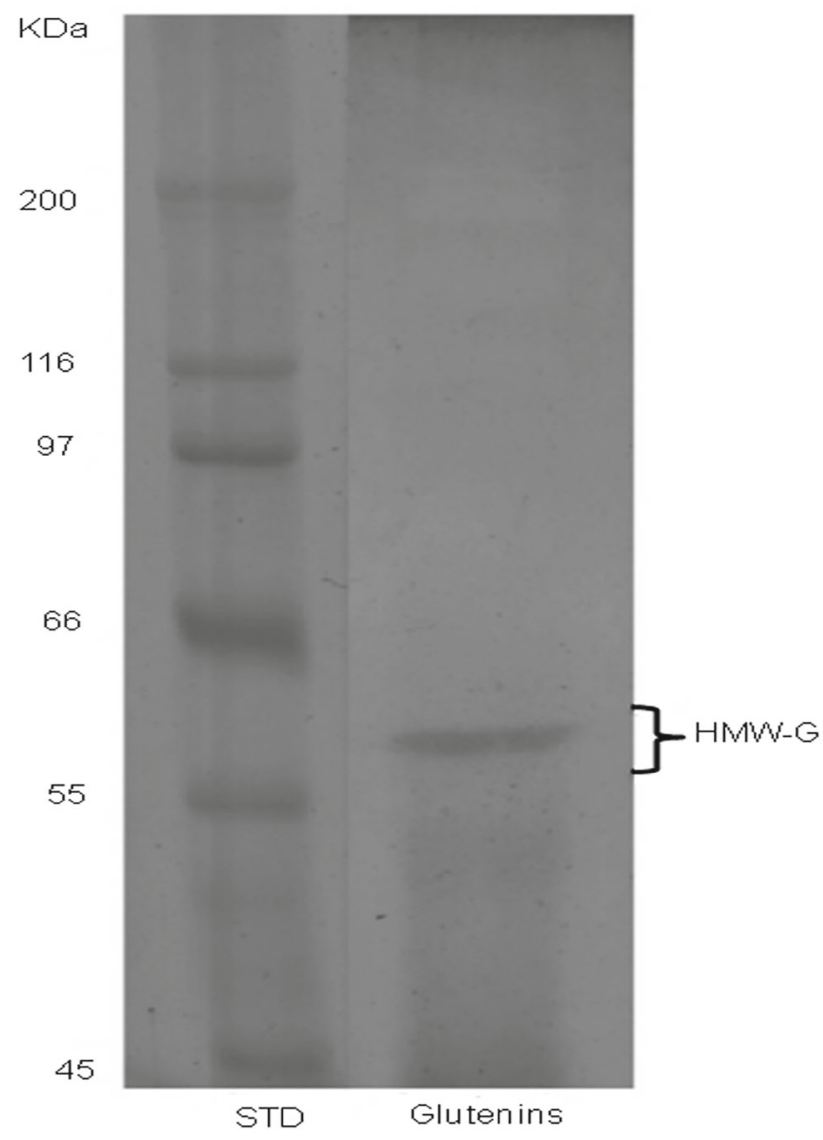

Figure 1. Electrophoretic profile of SFG.

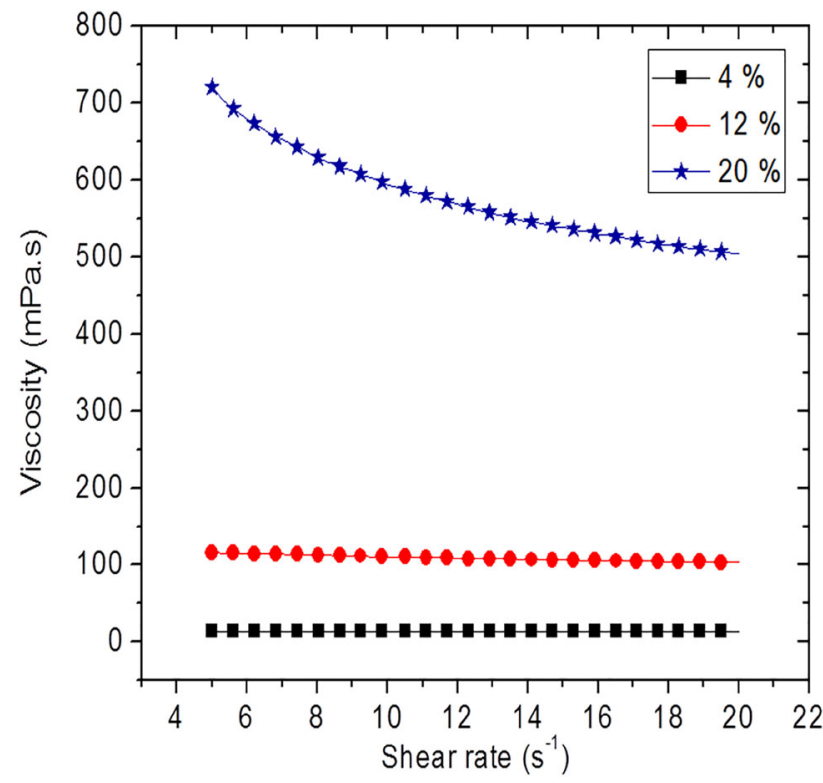

Figure 2. Dependence of viscosity of solutions of 4, 12 and $20 \%$ with respect to the shear rate.
Table 1. Type of fluid based on the power law.

\begin{tabular}{cccc}
\hline Solution SFG (\%) & Slope $(n)$ & $R^{2}$ & Type of fluid \\
\hline 4 & $0.99 \pm 0.00$ & 0.999 & Newtonian \\
12 & $0.91 \pm 0.07$ & 0.999 & Pseudoplastic \\
20 & $0.74 \pm 0.01$ & 0.999 & Pseudoplastic \\
\hline
\end{tabular}

increase in the concentration of solids in the solution [26]. In this case, viscosity decreases with the increase in shear rate (figure 2), due to the primary and secondary bonds present in the protein, which are isothermally and reversibly dissociated at a faster rate than the binding rate [27].

Figure 3 presents the effect of time on viscosity at a constant shear rate. In the $4 \%$ solution, an effect of time on viscosity is not present due to its Newtonian character. However, more concentrated solutions exhibited an increase in viscosity with time. This behaviour is known as rheopectic and can be attributed to two mechanisms that can occur in more concentrated solutions: (1) the dissociation rate of the polymer chains is slower than the rate of association, and (2) the low shear rate does not affect the breaking of links in the chain and only promotes the association of the chains [24]. Rheological analysis of the solutions indicates that the $4 \%$ solution is the best for obtaining particles, due to that viscosity is stable with respect to shear rate and time, since viscosity comprises a key factor to obtain particles with homogeneous sizes by the electrospray technique. On the other hand, all the solutions revealed viscosity values below the limit of viscosity $\left(800 \mathrm{mPa} \mathrm{s}^{-1}\right)$ to obtain particles. To verify the latter, we prepared particles from all the solutions by electrospray and particle size and morphology were analysed by SEM.

\subsection{Morphological studies}

Figure 4 presents the micrographs of glutenins and SFG powder (figure 4a, b). Morphological differences are observed in both the micrographs. Glutenins exhibit amorphous granules of different sizes (range 78-366 $\mu \mathrm{m}$ ), while with SFG, we observed amorphous flakes. The morphological change between both the samples can be attributed to a conformational change of the protein due to the SFG extraction process. This change can be observed by following the amide I band in the IR spectrum.

Particles of the 4, 12 and $20 \%$ solutions are illustrated in figure 5, where nano- and microparticles were obtained by electrospray. The nanoparticles obtained from the $4 \%$ concentration (figure 5a) were observed as agglomerated with sizes of $675 \mathrm{~nm}$ and uniform distribution; however, they did not present a defined morphology. This behaviour is probably due to the low concentration of SFG, causing insufficient intermolecular cross-links among the polymer chains, decreasing 


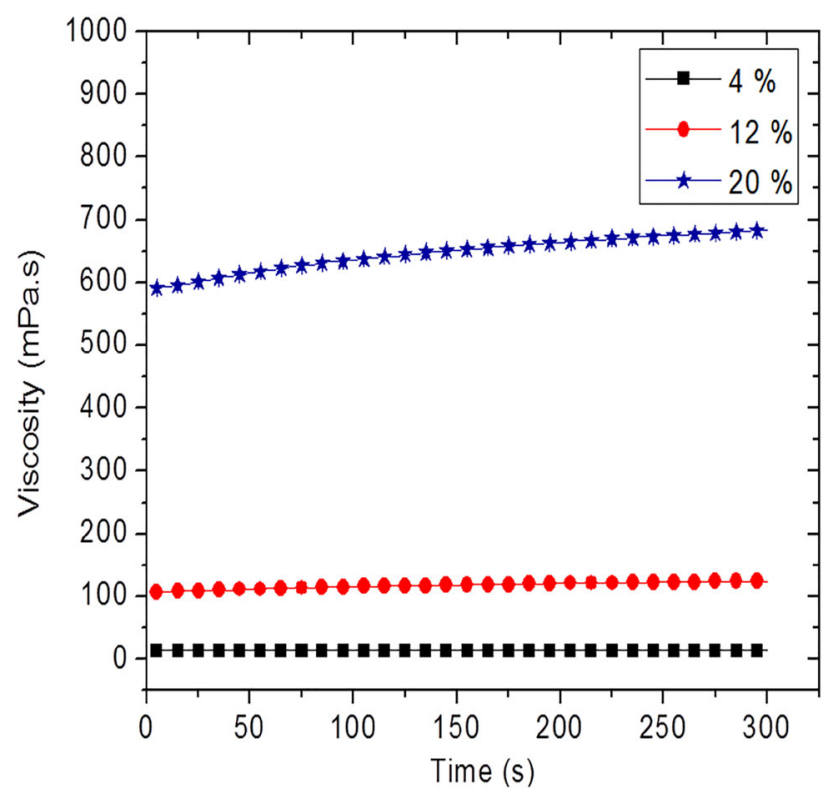

Figure 3. Effect of time on the viscosity of the solutions of 4, 12 and $20 \%$ at a constant shear rate of $5 \mathrm{~s}^{-1}$.
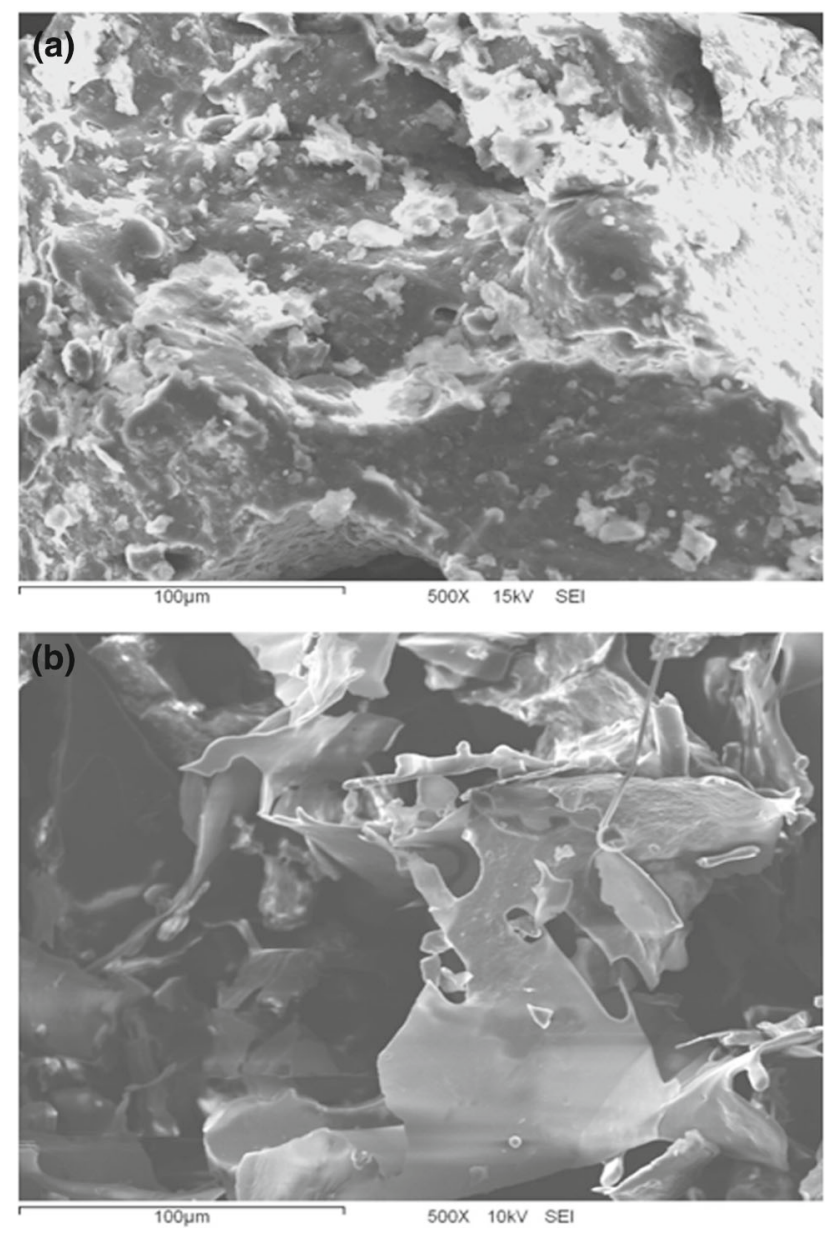

Figure 4. SEM micrographs: (a) wheat glutenins and (b) SFG. the ability to form defined particles after solvent evaporation by electrospray [15].

The $12 \%$ concentration (figure $5 \mathrm{~b}$ ) demonstrates particles with spherical morphology, and the sizes are smaller and larger than in the $4 \%$ concentration $(<600 \mathrm{~nm}$ to $>3 \mu \mathrm{m})$. This perfectly spherical morphology is attributed to the concentration of SDS, because it has been shown that SDS interacts with proteins and facilitates the formation of spherical structures at an adequate concentration. Turro et al [28] studied the interactions and complex structures formed from the bovine serum albumin protein and SDS by spectroscopic techniques, and the authors concluded that SDS and protein can form spherical structures depending on the concentration of SDS. Podaralla and Peumal [29] studied the influence of surfactants on the preparation of zein nanoparticles by nanoprecipitation. These authors utilized two types of surfactants: Pluronic F68 and lectin at different concentrations. The authors concluded that when the concentration of both surfactants increases, particle size decreases. In contrast, the $20 \%$ concentration (figure 5c) shows large and amorphous particles of various sizes.

This analysis suggests that based on what is seen in the literature and in the results obtained, that surfactants such as SDS can contribute significantly to the morphology and particle size from proteins. The diversity of sizes obtained in the particles of higher concentration (12 and 20\%) confirms that observed in the rheological analysis of the solutions, where viscosity was unstable for the both solutions. Based on what was observed in the SEM micrographs, we decided to select the $12 \%$ concentration microparticles to study their application as a controlled-release fertilizer. Thus, the latter was the concentration with which microparticles with best morphology were obtained, in that the release of compounds from amorphous particles can occur irregularly [30].

\subsection{Loading amount of urea}

Loading amount of urea in the glutenin microparticles was $88 \%$, containing $52.9 \mathrm{mg}$ of urea. This result is higher compared with other methods such as emulsion-based techniques, which involve the removal of particles from an aqueous phase. Wu et al [31] obtained microparticles of alginate sodium and chitosan, loaded with nitric oxide from a double emulsion (water-oil-water), reporting a loading efficiency of 64-70\%. This suggests that higher load efficiency could be obtained from the electrospray technique compared with the emulsion method. This result can be attributed to good solubility of urea in the solvent and the physical interaction between the two components [32].

\section{$3.5 \quad$ FT-IR analysis}

Figure 6 presents the glutenin (a) and SFG (b) spectra. In both the spectra, the characteristic bands of the proteins 

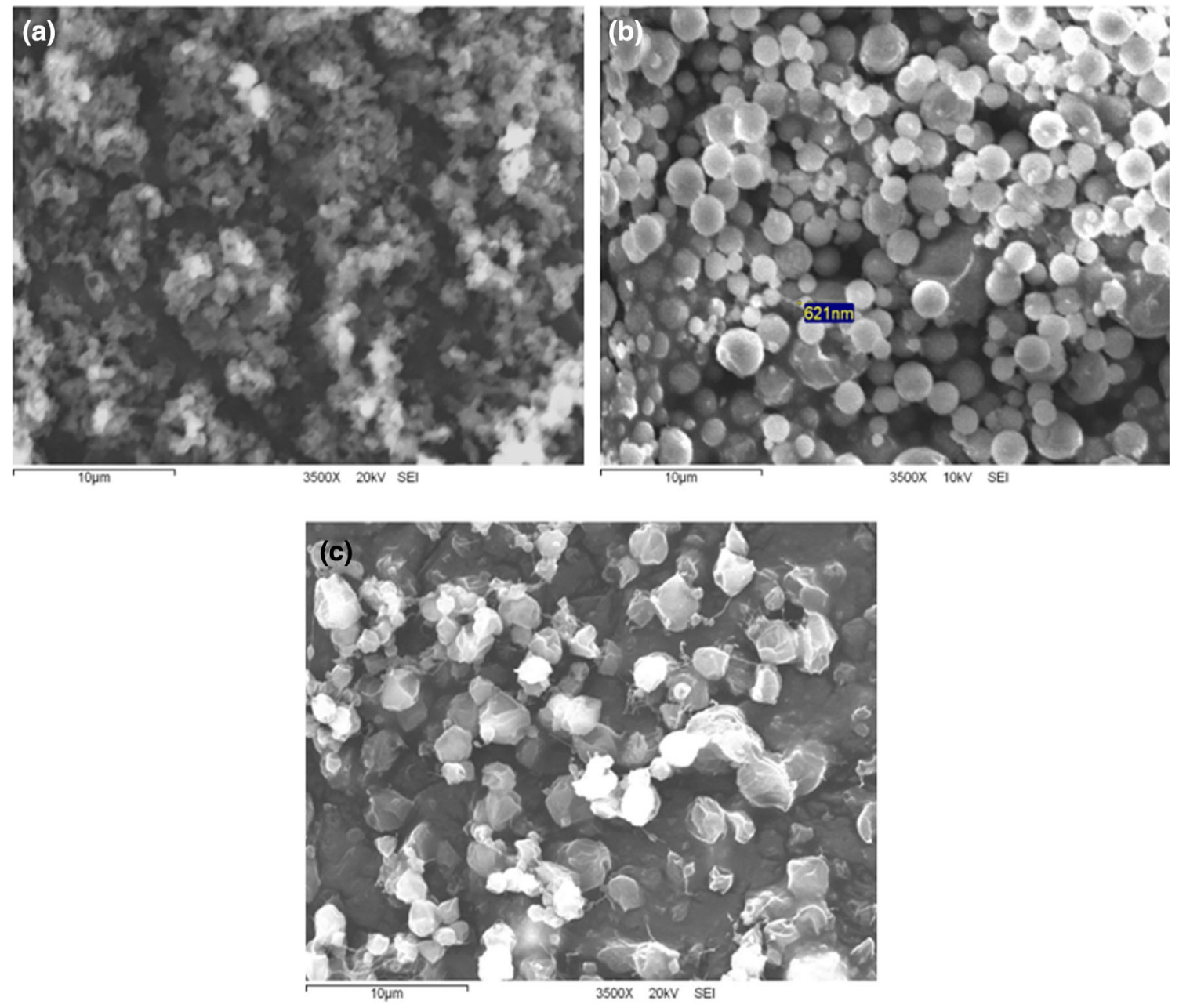

Figure 5. SEM micrographs of microparticles obtained by electrospray: (a) 4, (b) 12 and (c) 20\% solutions.

are observed, such as the intense $\mathrm{O}-\mathrm{H}$ stretch-related band observed in the $3400-3200 \mathrm{~cm}^{-1}$ region. The literature suggests that this band is related to the presence of water in proteins. Other important bands include the amide I band corresponding to the vibratory stretching of the group $\mathrm{C}=\mathrm{O}$, and amide II band, which is related to the stretching involved in $\mathrm{N}-\mathrm{H}$ deformation, which is in turn related to a change in the secondary structure of proteins $[12,33]$.

Comparing the glutenin and SFG spectra, a band shift in amide I $\left(1633-1651 \mathrm{~cm}^{-1}\right)$ and amide II $\left(1519-1531 \mathrm{~cm}^{-1}\right)$ bands at a greater wavelength, indicated by the dotted line is observed. This shift observed in the SFG spectrum in the amide I band is mainly related to a change in the secondary structure of the proteins [34]. The literature indicates that the presence of bands in the regions of 1640-1620 and $1695-1690 \mathrm{~cm}^{-1}$ is related to $\beta$-sheet, and the presence of bands between 1658 and $1654 \mathrm{~cm}^{-1}$ are assigned to $\alpha$-helix $[33,35,36]$. This indicates that in glutenins, there is a greater presence of the $\beta$-sheet and extracting the SFG and in turn reducing the hydrogen bonds present in the glutenins, gave rise to the protein acquiring the conformation of $\alpha$-helix.
This change can be confirmed with the morphology observed by SEM (figure $4 \mathrm{~b}$ ) for SFG. In addition, the $\mathrm{O}-\mathrm{H}$ band also exhibited a shift towards a greater wavelength (range $3278-3283 \mathrm{~cm}^{-1}$ ), probably by the reduction of hydrogen bonds on obtaining SFG.

Figure 7 depicts the spectra of the microparticles (a) and microparticles with urea (b). On comparing the spectra, two bands of primary amines are observed in the microparticles with urea in the regions of 3437 and $3345 \mathrm{~cm}^{-1}$. These bands are attributed to the presence of urea, which can be confirmed by the urea spectrum depicted in figure $7 \mathrm{c}$. In addition, amide I band of the microparticles with urea demonstrated a displacement of $30 \mathrm{~cm}^{-1}$ at a greater wavelength. This shift is attributed to the possible physical interaction between protein and urea by hydrogen bonds. This behaviour was reported in a previous work [20].

\subsection{Thermal analysis}

Table 2 presents the thermal stability of the glutenins, SFG and microparticles with and without urea. All samples revealed a 


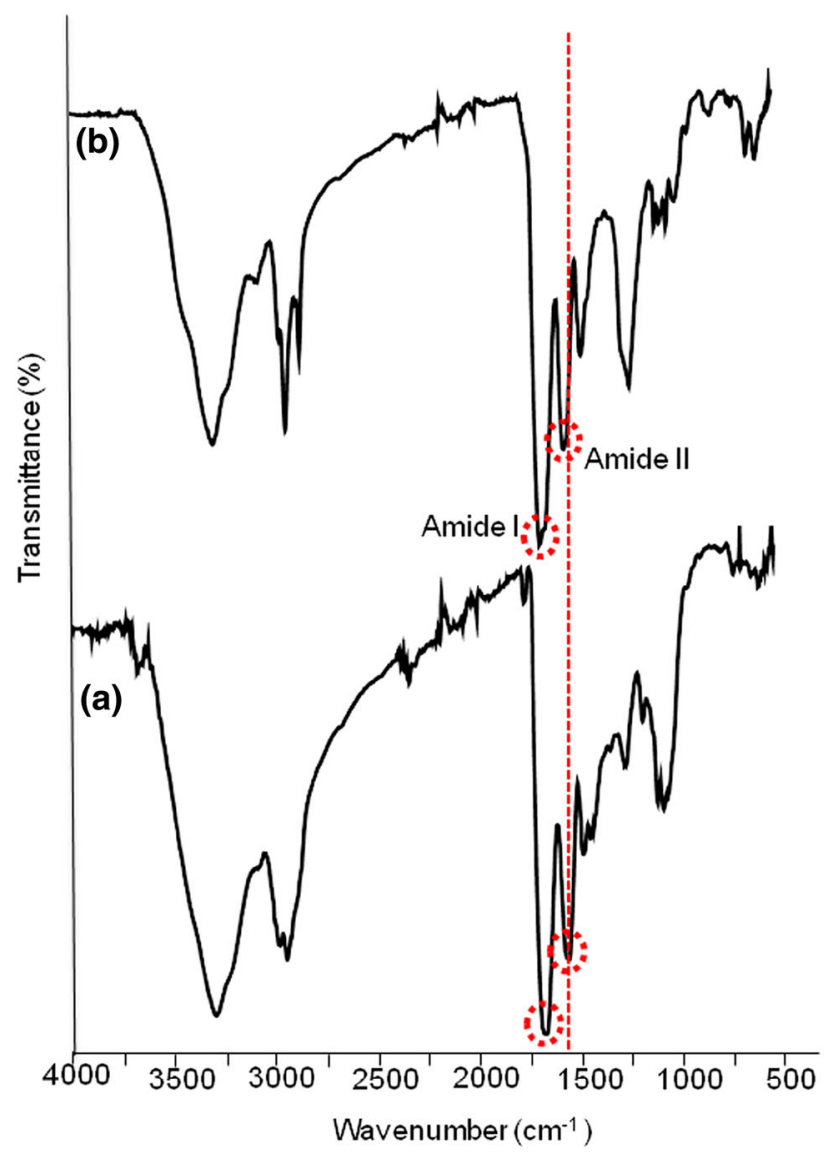

Figure 6. FT-IR spectra of (a) powdered glutenins and (b) SFG.

loss in weight at $100^{\circ} \mathrm{C}$ between 4 and $8 \%$, which is attributed to moisture. A second decrease is shown for the glutenins and SFG; in glutenins there is a weight loss of $51 \%$ at $202^{\circ} \mathrm{C}$, while for SFG, there is a $46 \%$ weight loss at $150^{\circ} \mathrm{C}$. The greater thermal stability of glutenins can be due to the structural conformation present in the protein. As already discussed in the FT-IR section, glutenins exhibited the greater presence of $\beta$-sheet, and the literature has reported that $\beta$-sheet structure is more stable than that of $\alpha$-helix [37]. The onset of degradation for both proteins is attributed to their fractionation in short, LMW chains [38], which are degraded totally at $700^{\circ} \mathrm{C}$. Moreover, the microparticles without urea demonstrated greater thermal stability compared to SFG; this increase in thermal stability is due to the presence of SDS $[39,40]$. According to the thermogram of SDS (not shown), SDS showed a thermal stability of around $200^{\circ} \mathrm{C}$, without being completely degraded.

This coincides with that partial degradation of microparticles with and without urea, leaves a residue of $16 \%$ in both cases. However, the microparticles with urea begin to degrade at $117^{\circ} \mathrm{C}$ with a mass loss of $16 \%$. This lower thermal stability as compared to microparticles without urea is attributed to the presence of urea, because urea has a thermal stability of $\sim 117^{\circ} \mathrm{C}$ [20]. This confirms the presence of urea

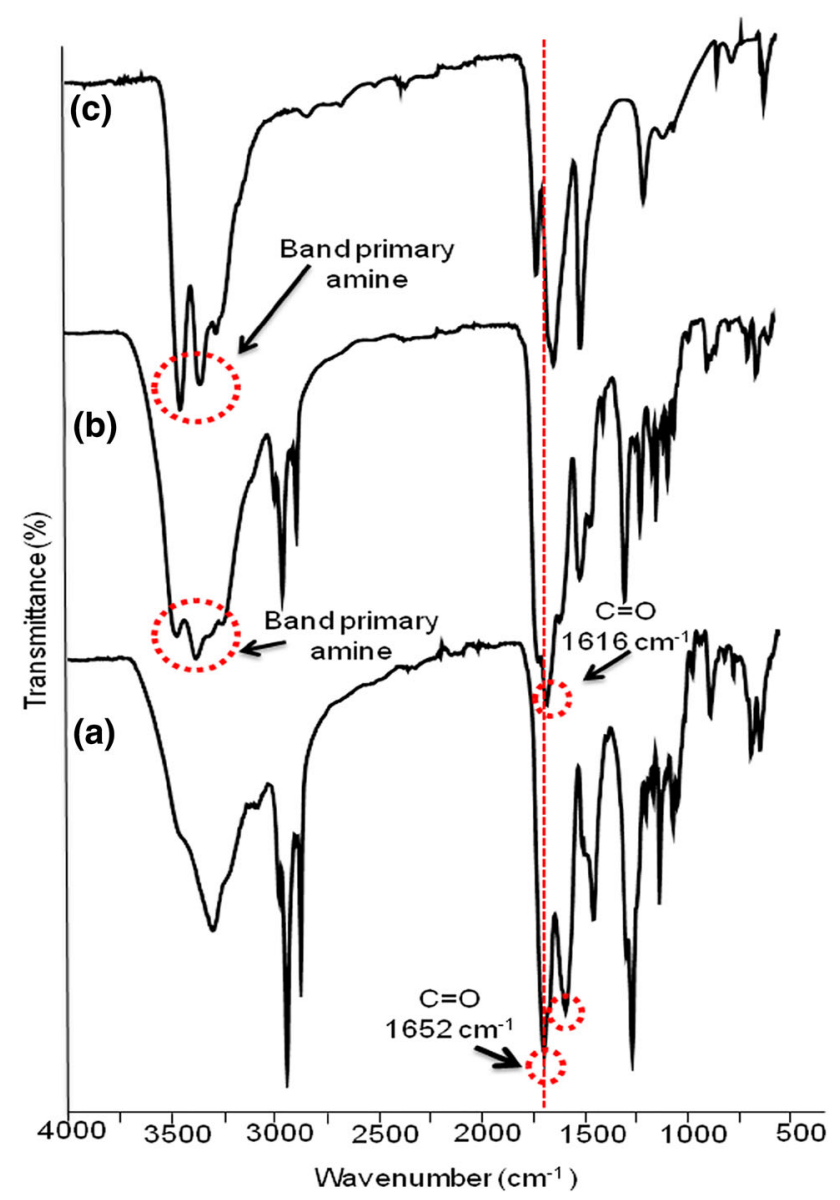

Figure 7. FT-IR spectra of (a) SFG microparticles $12 \%$, (b) SFG microparticles $12 \%$ with urea and (c) powdered urea.

in the microparticles with urea and is also observed by FT-IR. Hence, the microparticles with urea present a thermal stability of $117^{\circ} \mathrm{C}$ indicating that they can be applied to agricultural soils with temperatures above $100^{\circ} \mathrm{C}$ without being degraded by the temperature.

\subsection{Release kinetics of urea}

Figure 8 illustrates the release behaviour of urea at $\mathrm{pH} 4$, 7 and 10. During the first $30 \mathrm{~min}$ (figure 8b), a burst effect presents for all cases. This effect is associated with the presence of urea on the surface of the microparticles [17,41]. In figure $8 \mathrm{a}$ and at $\mathrm{pH} \mathrm{4}$, release behaviour is faster as compared with $\mathrm{pH} 7$ and 10 , reaching equilibrium in $4 \mathrm{~h}$. On increasing the $\mathrm{pH}$, the kinetic behaviour also changes, reducing the release time on reaching equilibrium at $5(\mathrm{pH} 7)$ and $12 \mathrm{~h} \mathrm{(pH} \mathrm{10),} \mathrm{releasing} \mathrm{99 \%} \mathrm{of} \mathrm{urea} \mathrm{in} \mathrm{all} \mathrm{cases.} \mathrm{Proteins,}$ due to their amphoteric character, can respond to changes in $\mathrm{pH}$ through the carboxylic acid groups and amino groups present in the structure. Therefore, the behaviour of the kinetics of the release of urea can be attributed to ionization of the amino groups when the $\mathrm{pH}$ is acidic, dissociating the hydrogen bonds that formed between the protein and urea, causing 
Table 2. Thermal stability obtained by TGA.

\begin{tabular}{lcc}
\hline \multirow{2}{*}{ Sample } & $\begin{array}{c}\text { Temperature } \\
\left({ }^{\circ} \mathrm{C}\right)\end{array}$ & $\begin{array}{c}\text { Weight loss } \\
(\%)\end{array}$ \\
\hline Glutenin powder & 100 & 6 \\
& $202-368$ & 51 \\
FSG & $381-700$ & 43 \\
& 100 & 5 \\
Microparticles of FSG & $150-336$ & 46 \\
& $350-700$ & 49 \\
& 100 & 4 \\
Microparticles of FSG with urea & $175-262$ & 25 \\
& $262-336$ & 21 \\
& $346-700$ & 34 \\
& 100 & 8 \\
& $117-208$ & 16 \\
& $225-266$ & 14 \\
& $271-328$ & 20 \\
& $343-700$ & 26 \\
\hline
\end{tabular}

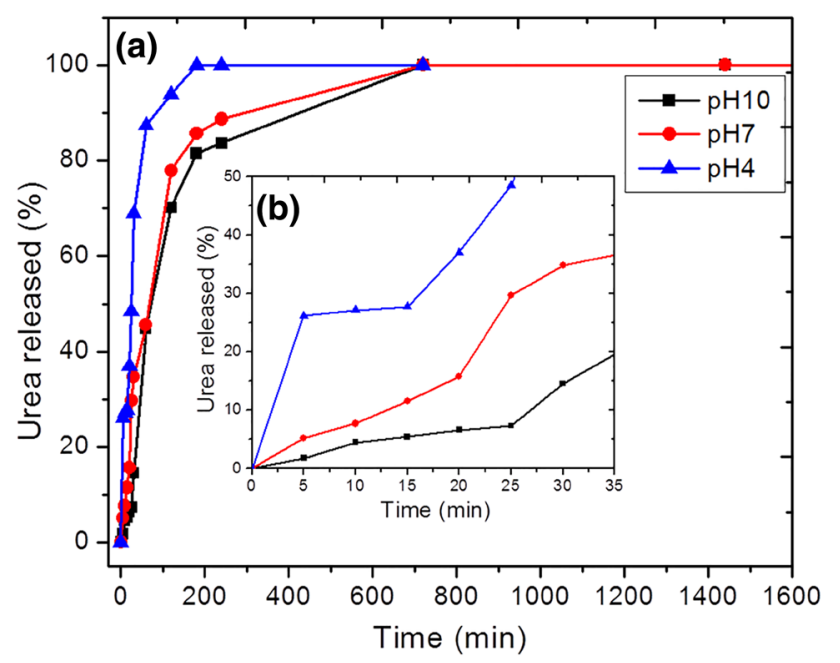

Figure 8. Effect of $\mathrm{pH}$ on percentage of release of urea with respect to time: $\mathrm{pH} \mathrm{4,} 7$ and $10(\mathrm{a}, \mathrm{b})$.

rapid release. This is due to the effect of the non-ionization of carboxylic acid $(-\mathrm{COOH})$ and the ionic form of the amino group $\left(\mathrm{NH}_{3}^{+}\right)[3,42]$. If there is an alkaline medium $(\mathrm{pH} 10)$, the carboxylic acid passes to its ionic form (-COO-) and the amino group, to its non-ionic form $\left(\mathrm{NH}_{2}\right)$, allowing the formation of hydrogen bonds, decreasing the release of urea into the medium [43]. However, in a neutral medium $(\mathrm{pH}$ 7 ), there can be a ratio of ionized groups and non-ionized groups, giving rise to a decrease in the formation of interactions via hydrogen bonding [44]. These results are promising since, throughout the experiment, the tablets were in contact with the solution buffers, and time-of-release until equilibrium was of hours. Therefore, release is expected to be longer in soil.
Table 3. Data obtained from the equation of Ritger and Peppas.

\begin{tabular}{rcccc}
\hline $\mathrm{pH}$ & $R^{2}$ & $k$ & $n$ & Mechanism \\
\hline 4 & 0.993 & 0.365 & 1.0 & Transport case II \\
7 & 0.999 & 0.329 & 1.0 & Transport case II \\
10 & 0.992 & 0.251 & 0.5 & Diffusion \\
\hline
\end{tabular}

\subsection{Empirical model}

Table 3 lists the values obtained for $k$ and $n$ from the model of Ritger and Peppas. The value of $k$ at $\mathrm{pH} 4$ is higher than at $\mathrm{pH} 7$ and 10 . The kinetic constant $(k)$ is related to the release rate, whereby the values obtained for each $\mathrm{pH}$ match the kinetic behaviour of the urea, as discussed previously. The mechanisms involved in the release of urea included the transport of case-type II at $\mathrm{pH} 4$ and 7, indicating that the release of urea is accomplished by matrix swelling, while for $\mathrm{pH} 10$, it is carried out by a diffusion process [12].

\section{Conclusions}

Viscosity and fluid type of the solutions play an important role in obtaining particles by the electrospray technique. In addition, Newtonian fluids are suitable to obtain particles that are homogeneous in size. It was observed that the use of concentration-dependent SDS can comprise a factor in the morphology of particles from proteins. However, we suggest, for future works, carrying out of particles from solutions at different protein and SDS concentrations for a better study. The FT-IR spectra of glutenins and SFG revealed changes in their secondary structure. Also, it was observed that microparticles with urea obtained by electrospray exhibited physical interactions with urea and thermal stability $>100^{\circ} \mathrm{C}$. The mechanisms of urea release and rate depend largely on the $\mathrm{pH}$ of the medium, reaching a release of up to $12 \mathrm{~h}$. Therefore, with these results, we can conclude that glutenin microparticles are a promising material for use as a controlled-release fertilizer in agricultural soils. In addition, due to the good characteristics observed, glutenin microparticles can be applied in the release of various compounds such as pharmaceuticals, antioxidants, colourants and others.

\section{Acknowledgements}

We acknowledge the University of Sonora and appreciate the economic support of CONACyT through the Basic Science Project number 178436.

\section{References}

[1] Hernández P, Villalobos R and Chiralt A 2004 Food Hydrocolloids $\mathbf{1 8} 403$ 
[2] Robles M, Rodríguez F, Márquez E, Barrera A, Aguilar J and Del Toro C 2014 Biotecnia 1644

[3] Reddy N and Yang Y 2011 Trends Biotechnol. 29490

[4] Kayserilioğlu B, Bakir U, Yilmaz L and Akkaş N 2003 Bioresour. Technol. 87239

[5] Lagrain B, Rombouts I, Delcour J and Koehler P 2014 J. Cereal Sci. 6131

[6] Díaz P, Dalla M, Vázquez D and Castro M 2006 Agric. Técnica 66360

[7] Song Y and Zheng Q 2008 J. Cereal Sci. 4877

[8] Chen L, Reddy N, Wu X and Yang Y 2012 Ind. Crops Prod. 35 70

[9] Xu H, Cai S, Sellers A and Yang Y 2014 J. Biotechnol. 184 179

[10] Reddy N, Shi Z, Xu H and Yang Y 2015 J. Biomed. Mater. Res. A 1031653

[11] Davoodi P, Feng F, Xu Q, Yan W, Tong Y, Srinivasan M et al 2015 J. Control. Release 20570

[12] Andreani L, Cercená R, Ramos B and Soldi V 2009 Mater. Sci. Eng. C 29524

[13] Lee Y, Mei F, Bai M, Zhao S and Chen D 2010 J. Control. Release 14558

[14] Jaworek A and Sobczy A 2008 J. Electrostat. 66197

[15] Gómez J, Balaguer M, Gavara R and Hernandez P 2012 Food Hydrocolloids 2882

[16] Xu Q, Qin H, Yin Z, Hua J, Pack D and Wang C 2013 Chem. Eng. Sci. 104330

[17] Zarchi A, Abbasi S, Faramarzi M, Gilani K, Ghazi M and Amani A 2015 Int. J. Biol. Macromol. 72764

[18] Jaworek A 2007 Powder Technol. 17618

[19] Lagrain B, Goderis B, Brijs K and Delcour J 2010 Biomacromolecules 11533

[20] Castro D, Rodríguez F, Ramírez B, Torres P, Castillo M, Rodríguez D et al 2012 Materials 52903

[21] Chime S, Onunkwo G and Onyish I 2013 Res. J. Pharm. Biol. Chem. Sci. 497

[22] Gianibelli M and Solomon R 2003 J. Cereal Sci. 37253

[23] Chakraborty S, Liao I, Adler A and Leong K 2009 Adv. Drug Deliv. Rev. 611043
[24] Triantafillopoulos N 1998 Measurement of fluid rheology and interpretation of rheograms manual (Michigan, USA: Kaltec Scientific Inc.) 2nd edn. p 21

[25] Dong J, Asandei A and Parnas R 2010 Polymer 51 3164

[26] Chhabra R P 2010 Non-Newtonian fluids: an introduction In Rheology of complex fluids (New York, NY: Springer) p 3

[27] El Miri N, Abdelouahdi K, Barakat A, Zahouily M, Fihri A, Solhy A et al 2015 Carbohydr. Polym. 129156

[28] Turro N, Lei X, Ananthapadmanabhan K and Aronson M 1995 Langmuir 112525

[29] Podaralla S and Perumal O 2012 AAPS PharmSciTech 13 919

[30] Prabhakaran M, Zamani M, Felice B and Ramakrishna S 2015 Mater. Sci. Eng. C 5666

[31] Wu W, Gaucher C, Fries I, Hu X, Maincent P and Sapin-Minet A 2015 Int. J. Pharm. 495354

[32] Xu Y and Hanna M 2006 Int. J. Pharm. 32030

[33] Li W, Dobraszczyk B, Dias A and Gil A 2006 Cereal Chem. 83 407

[34] Barth A 2007 (BBA)-Bioenerg. 91073

[35] Kong J and Yu S 2007 Biochim. Biophys. Sin. 39549

[36] Balaguer M, Borne M, Chalier P, Gontard N, Morel M, Peyron S et al 2013 Biomacromolecules 141493

[37] Perczel A, Gáspári Z and Csizmadia I 2005 J. Comput. Chem. 261155

[38] Wang P, Xu L, Nikoo M, Ocen D, Wu F, Yang N et al 2014 Food Hydrocolloids 35238

[39] Hazarika J and Kumar A 2013 Synth. Met. 175155

[40] Jiang D, Yao Q, McKinney M and Wilkie C 1999 Polym. Degrad. Stab. 63423

[41] Gulfam M, Kim J, Lee J, Ku B, Chung B and Chung B 2012 Langmuir 288216

[42] Chen L, Remondetto G and Subirade M 2006 Trends Food Sci. Technol. 17272

[43] Rodriguez D, Perez C, Castillo M, Perez M, Romero J, Ledezma A et al 2012 Polym. Bull. 68197

[44] Farooq U, Khan M, Athar M and Kozinski J 2011 Chem. Eng. J. 171400 